\title{
Anólise eletromiográfica durante sessões de equoterapia em praticantes com paralisia cerebral
}

\author{
Electromyographic analysis during hippotherapy sessions in \\ practitioners with cerebral palsy
}

\author{
Ana Paula Espindula'; Mayara Simões²; Iramaia Salomão Alexandre de Assis's; Mariane \\ Fernandes; ${ }^{3}$ Alex Abadio Ferreira4; Patrícia Fonseca Ferraz; Isabella Cardoso Cunha6; \\ Mara Lúcia da Fonseca Ferraz; Luciane Aparecida Pascucci Sande de Souza ${ }^{8}$; Vicente de Paula \\ Antunes Teixeira ${ }^{9}$ \\ ${ }^{1}$ Doutora em Ciências da Saúde, área de concentração Patologia Humana, Professora do curso de Fisioterapia - UFTM; Fisioterapeuta \\ na - APAE. Uberaba, MG - Brasil. \\ ${ }^{2}$ Graduandas em Fisioterapia - UFTM. Uberaba, MG - Brasil. \\ Mestranda em Ciências da Saúde, área de concentração Patologia Geral - UFTM. Fisioterapeuta - APAE. Uberaba, MG - Brasil \\ ${ }^{4}$ Coordenador Clínico e Fisioterapeuta - APAE. Uberaba, MG - Brasil. \\ ${ }^{5}$ Graduanda em Engenharia Ambiental - Uniube. Uberaba, MG - Brasil. \\ ${ }^{6}$ Graduanda em Ciências Biológicas - UFTM. Uberaba, MG - Brasil. \\ Doutora em Ciências da Saúde, área de concentração Patologia Geral, e Bióloga - UFTM. Uberaba, MG - Brasil. \\ ${ }^{8}$ Doutora em Biologia Funcional e Molecular - Unicamp; Professora do curso de Fisioterapia - UFTM. Uberaba, MG - Brasil. \\ ${ }^{9}$ Doutor em Ciências da Saúde, área de concentração Patologia Geral, Professor Titular da Disciplina de Patologia Geral - UFTM. \\ Uberaba, MG - Brasil. \\ Endereço de correspondência \\ Ana Paula Espindula \\ R. Frei Paulino, 30 \\ 38025-180 - Uberaba, MG - Brasil \\ anapaulaespindula@yahoo.com.br
}

\begin{abstract}
Resumo
A equoterapia destaca-se como forma de tratamento para indivíduos com Paralisia Cerebral (PC). Objetivo: Verificar o efeito do tipo de montaria na atividade muscular em portadores de PC. Métodos: Avaliaram-se três praticantes com PC por eletromiografia, durante quatro sessões de equoterapia, utilizando-se, em cada sessão, uma condição de montaria. Realizou-se análise estatística descritiva com valores de média e desvio-padrão dos RMS normalizado. Resultados: O músculo trapézio esteve sempre ativo, nas posições sentado inicial e final, em todas as condições de montaria, exceto na sela sem apoio dos pés. Nos três momentos avaliados durante as sessões, observa-se que, nas montarias com manta, a atividade do trapézio também foi alta, comparada com a dos demais músculos. O apoio dos pés permitiu ativação mais homogênea dos músculos de tronco avaliados, quando comparado à ausência do apoio. Conclusão: Usar sela com pé no estribo foi a melhor opção para tratamento equoterapêutico dos hemiparéticos avaliados.

Descritores: Eletromiografia; Equoterapia; Paralisia Cerebral.
\end{abstract}

\begin{abstract}
Introduction: Hippotherapy has emerged as a form of treatment for individuals with Cerebral Palsy (CP). Objective: To verify the effect of the mount type in the muscular activity in patients with $\mathrm{CP}$. Methods: Three practitioners with $\mathrm{CP}$ were evaluated by electromyography during four hippotherapy sessions. In each session the mount condition was changed. A descriptive statistical analysis with mean and standard deviation of normalized RMS was performed. Results: The trapezius muscle was always active in the positions initial sitting and final sitting, in all mount conditions, except in the saddle without the feet support. At the three moments evaluated during sessions, it was observed in the condition horse with blanket, that muscle activity was also high in trapezius compared to the other muscles. The feet support allowed a more homogeneous activation of the trunk muscles evaluated, when compared to lack of support. Conclusion: To use saddle on foot in the stirrup was the best option for the hippotherapy treatment of the hemiparetic individuals evaluated.
\end{abstract}

Key words: Cerebral Palsy; Electromyography; Hippotherapy. 


\section{Introdução}

O termo Paralisia Cerebral (PC) é descrito na literatura como um grupo de desordens permanente do desenvolvimento, do movimento e da postura, causando limitações que são atribuídas a um distúrbio não progressivo que ocorre durante o desenvolvimento cerebral fetal ou infantil. Os distúrbios motores da PC são frequentemente acompanhados de perturbações na sensibilidade, na percepção, na cognição, na comunicação, no comportamento, além de epilepsia e problemas musculoesqueléticos secundários ${ }^{1}$. No Brasil, os dados estimam cerca de 30 a 40 mil novos casos por ano ${ }^{2,3}$ dessa anomalia.

As sequelas neurológicas são persistentes, porém não variáveis, e produzem uma incapacidade primária, como distinção característica das alterações e desenvolvimento motor normal dos reflexos primitivos, tônus e controle postural, e provoca déficits dos padrões de movimentos e posturas anormais ${ }^{4,5}$. Condições secundárias, como distúrbios associados às alterações sensitivas, perceptuais e cognitivas, podem existir e influenciar nas habilidades motoras e na compreensão, bem como numa ampla variedade de transtornos musculoesqueléticos ${ }^{6-11}$.

A classificação clínica da paralisia cerebral ocorre mediante a avaliação do tônus muscular, sendo classificada como espástica, quando ocorre um aumento do tônus de base; hipotônica, quando há diminuição do tônus de base; atetósica e atáxica. Quanto ao comprometimento dos esqueletos apendiculares, é feito ainda outra classificação topográfica em: tetraperesia, diparesia ou hemiparesia ${ }^{12}$. Somado a isso, o diagnóstico de PC depende também do período em que ocorreu e da extensão das lesões cerebrais, assim como do quadro clínico apresentado ${ }^{11,13}$.

A forma espástica é a mais frequente. Nela, encontram-se hipertonia muscular extensora e adutora dos membros inferiores, hiperreflexia profunda e sinal de Babinski, déficit de força localizado ou generalizado, dependendo da extensão do comprometimento ${ }^{14}$. A persistência do aumento do tônus muscular origina posturas anormais que, geralmente, tornam-se típicas da criança e podem converter-se em deformidades ou contraturas $^{15}$. Considerando a topografia associada, o indivíduo com hemiparesia espástica comumente apresenta resistência e limitação da amplitude de movimento, déficit postural, de tônus e força muscular, encurtamento musculares e atrofia do membro superior e inferior acometido ${ }^{16,17}$.

Dentre as várias opções de tratamento para os casos de hemiparesia espástica existe a equoterapia, um método terapêutico e educacional que utiliza o cavalo em uma abordagem interdisciplinar. Nessa terapia, o indivíduo recebe o movimento do cavalo, semelhante ao do ser humano, estimulando a sua movimentação ativa por meio dos movimentos do animal ${ }^{18}$.

Na literatura, encontram-se alguns estudos utilizando a equoterapia em crianças com PC, em que se descrevem melhora no controle postural - por meio da estimulação das reações de equilíbrio normais e por meio de estímulos repetitivos de coordenação da postura - e na flexibilidade da cadeia muscular posterior, durante a sessão de equoterapia; relacionando, assim, a importância desse tipo de terapia nas assimetrias motoras presentes nesses indivíduos ${ }^{19-21}$.

Assim, considerando o potencial cinesioterapêutico produzido pela riqueza de estímulos desencadeados pelo movimento do cavalo ${ }^{22,23}, \mathrm{o}$ objetivo neste estudo foi verificar o melhor material de montaria para o recrutamento da musculatura de tronco de crianças com paralisia cerebral do tipo hemiparesia espástica.

\section{Material e métodos}

Este estudo foi avaliado e aprovado pelo Comitê de Ética e Pesquisa (CEP) da Universidade Federal do Triângulo Mineiro UFTM, com protocolo de $\mathrm{n}^{\circ} 1502$.

\section{Participantes}

Como critério de inclusão, os praticantes com diagnóstico de PC deveriam ter enca- 
minhamento médico para a prática da equoterapia; e o responsável ter assinado o Termo de Responsabilidade, que autoriza essa prática. Como critério de exclusão, os pacientes não poderiam apresentar epilepsia não controlada, comportamento autodestrutivo ou medo incoercível, instabilidades da coluna vertebral, luxações de ombro ou de quadril, escoliose em evolução de 30 graus ou mais, hidrocefalia com válvula. Inicialmente, foram selecionadas 12 crianças, conforme organograma (Figura 1).

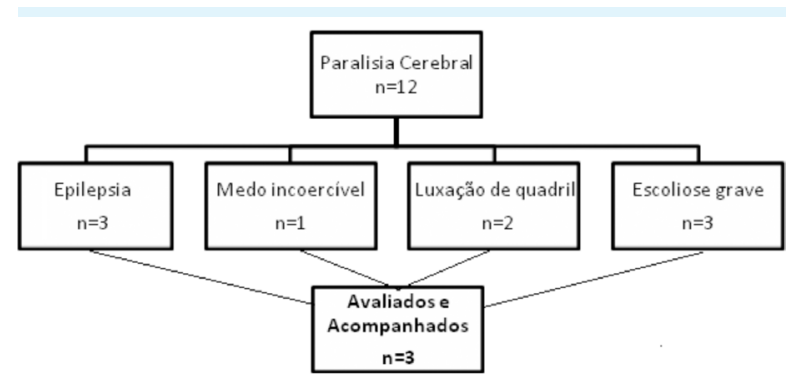

Figura l: Organograma representativo dos motivos de exclusão e desistência dos sujeitos da pesquisa

Participaram de todas as fases deste estudo três praticantes do gênero masculino, com diagnóstico clínico de paralisia cerebral e fisioterapêutico de hemiparesia espástica leve, apresentado um a esquerda, e dois, a direita; com presença de padrão flexor em membro superior e extensor em membro inferior (Tabela 1). Esses alunos voluntários estavam regularmente matriculados na Associação de Pais e Amigos dos Excepcionais de Uberaba, MG (APAE).

Tabela 1: Dados antropométricos e funcionais da amostra

\begin{tabular}{crrrrr}
\hline Indivíduo & Idade & Altura & Peso & IM & Nível - \\
\hline 1 & 23 & 1,72 & 79 & 26,77 & \\
2 & 17 & 1,64 & 62 & 23,13 & \\
3 & 13 & 1,6 & 51 & 19,92 & \\
& & & & & \\
& 17,66 & 1,65 & 70,75 & 23,27 & \\
& 7,19 & 0,06 & 14,1 & 3,42 & \\
\hline
\end{tabular}

\section{Procedimentos de avaliação}

Para o registro da atividade elétrica muscular, foi utilizado, durante a sessão de equoterapia, um eletromiógrafo de superfície da marca EMG System do Brasil, de oito canais, 14 bits de resolução na aquisição de sinais, isolamento elétrico de 5000 volts, capacidade de aquisição de 2 mil amostras/segundo/canal, conectado a um notebook, via porta USB, e alimentado por bateria recarregável níquel-metal-hidreto, que não polui a natureza.

Inicialmente, foi realizada tricotomia e a limpeza do local com algodão e álcool 70\%, para eliminar ou diminuir os riscos de interferências nos eletrodos durante a captação dos sinais. Posteriormente, colocaram-se os eletrodos de superfície, autoadesivos, em pares, com distância de um eletrodo ao outro de três centímetros (optou-se por não diminuir o tamanho dessa distância intereletrodo, conforme sugere a International Society Electromyography and Kinesilogy (ISEK) para evitar diminuição da aderência dos eletrodos na pele dos voluntários), sobre os seguintes músculos bilateralmente: trapézio/fibras superiores (linha média entre o acrômio e C7), paravertebral (3 $\mathrm{cm}$ do processo espinhosos de T9), multífico ( $3 \mathrm{~cm}$ laterais ao nível do processo espinhoso de L5), reto abdominal ( $3 \mathrm{~cm}$ laterais da cicatriz umbilical) e o eletrodo de referência foi posicionado no punho esquerdo, sobre o processo estiloide da ulna, segundo as recomendações do projeto Surface Electromyography for Non-Invasive Assessment of Muscle-SENIAM ${ }^{24,25}$.

A atividade eletromiográfica está apresentada em valores da raiz quadrada da média (RMS), registrados durante a coleta e divididos pela contração voluntária máxima (CVM) do músculo avaliado. No teste de CVM do trapézio superior, os indivíduos foram posicionados em decúbito ventral, com os membros inferiores fixos e a cabeça imobilizada, recebendo resistência na região occipital enquanto realizavam uma extensão da cervical. Para avaliar os paravertebrais torácicos /eretores da espinha, o posicionamento foi o mesmo e os indivíduos apenas mantinham a extensão de tronco contra a gravidade. Da mesma forma, o teste dos multífidos foi realizado nessa posição aplicando-se resistência na parte superior do 
dorso em sentido oposto a extensão do tronco. Já, para testar os retos abdominais, os indivíduos foram posicionados em supino, com os membros inferiores em flexão e apoiados pelo terapeuta. Os indivíduos exerciam flexão de tronco elevando as escápulas do apoio, recebendo uma resistência no esterno.

Foram realizadas três repetições de CVM, e o valor médio foi utilizado para normalização dos dados. O valor obtido foi então a raiz quadrada da média normalizada (RMSn): RMSn=RMS/ CVM.

Os registros eletromiográficos foram realizados uma única vez em cada uma das etapas apresentadas a seguir (Tabela 2). As coletas nos momentos (M1, M2 e M3), que correspondem ao primeiro, décimo quinto e trigésimo minutos de sessão, respectivamente, foram realizadas com o cavalo na andadura, a passo.

Tabela 2: Momentos de coleta dos dados eletromiogróficos

\begin{tabular}{lc}
\hline \multicolumn{2}{c}{ Momentos e Tempos } \\
\hline Posição ortostática, antes da sessão & POI \\
Sentado no cavalo, com esse parado no início da sessão & SI \\
1' de sessão & M1 \\
$15^{\prime}$ de sessão & M2 \\
$30^{\prime}$ de sessão & M3 \\
Sentado no cavalo, com esse parado no final da sessão & SF \\
Posição ortostática, após sessão & POF \\
\hline
\end{tabular}

\section{Intervenção}

As sessões foram realizadas no Centro de Equoterapia da Associação de Pais e Amigos dos Excepcionais (APAE) de Uberaba, utilizando terrenos irregulares como percurso durante as sessões para um maior número de ajustes tônicos, o redondel para estimular o labirinto e ações de stop and go para provocar reação de equilíbrio, proteção e correção postural.

Cada praticante foi submetido a quatro sessões de equoterapia de 30 minutos, uma vez por semana, e em cada sessão utilizaram-se diferentes materiais de montaria e posicionamento dos pés (Tabela 3).
Tabela 3: Material de montaria utilizado nas sessões

\begin{tabular}{lc}
\hline \multicolumn{1}{c}{ Materiais utilizados } & Sessões \\
\hline Sela Australiana com posicionamento dos pés no estribo & $1^{\mathrm{a}}$ \\
Sela Australiana sem estribo & $2^{\mathrm{a}}$ \\
Manta de pano com posicionamento dos pés no estribo & $3^{\mathrm{a}}$ \\
Manta de pano sem estribo & $4^{\mathrm{a}}$ \\
\hline
\end{tabular}

Foi utilizado um cavalo treinado para a prática da equoterapia da raça Árabe que apresentava comportamento dócil, obediência à voz, não se perturbava com ruídos, encostavase na rampa de acesso dos indivíduos, gostava de crianças e aceitava todos os tipos de arreamento, com altura de $1,48 \mathrm{~cm}$ e idade de 20 anos. Possuía as três andaduras regulares: o galope, o trote e o passo, e na andadura a passo transpistava, sobrepistava e antepistava. Para realização deste estudo, foi utilizada a andadura a passo, com as variáveis transpistar, sobrepistar e antepistar, aleatórias durante os 30 minutos de sessão.

\section{Anólise dos dados}

Foi elaborada uma planilha eletrônica por meio do programa Microsoft Excel ${ }^{\circledR}$ e, a seguir, utilizada a estatística descritiva com valores de média das RMSn.

\section{Resultodos}

Os resultados estão apresentados na Tabela 4, com valores obtidos nas tarefas SI e SF e, na Tabela 5, com os valores encontrados nos momentos M1, M2 e M3. Nota-se que o músculo trapézio/fibras superiores esteve sempre muito ativo, especialmente em SI e SF, em todas as condições, exceto na sela com pés fora do estribo. Também nos momentos M1, M2 e M3 observa-se, para as condições em que foram utilizadas a manta, que a atividade do músculo trapézio/fibras superiores foi alta, comparado com a dos demais músculos. Verifica-se também, que em algumas situa- 
ções, os músculos tiveram sua ativação igual ou superior a CVM. Sendo estes: músculo trapézio superior bilateralmente, na tarefa 1 , condição 1 ; trapézio superior esquerdo, na tarefa 1, condição 2; paravertebral direito, na tarefa 2, condição 3 , e músculo abdominal esquerdo, na tarefa 1 e 2 , na condição 4 .

Nas Figuras 2 e 3, podem ser observados os resultados da atividade muscular normalizada nas seguintes condições: sela com apoio dos pés e sela sem apoio dos pés, respectivamente. De modo comparativo, nota-se que o apoio dos pés permitiu uma ativação mais homogênea entre os músculos avaliados, quando comparado à ausência do apoio. Entre os momentos (M1 a M3), os músculos multífidos esquerdo e reto abdominal esquerdo apresentaram grande variabilidade na condição sem apoio dos pés. Nesta também se destaca a alta atividade do músculo reto abdominal.

Na Figura 4, estão representados os resultados encontrados na condição sela com apoio dos pés nos estribos para uma melhor visualização do ganho na ativação de todos os músculos após a realização de uma sessão.

\section{Discussão}

A partir do objetivo proposto neste estudo, que foi verificar o melhor material de montaria para o recrutamento da musculatura de tronco na hemiparesia espástica, pode-se concluir que o uso da sela com apoio dos pés nos estribos garantiu uma ativação muscular mais homogênea e um ganho ao final da sessão.

Nas condições em que a manta foi utilizada, obteve-se uma maior ativação muscular na região cervical (trapézios fibras superiores). Esse achado mostra o impacto de uma possível sobrecarga nessa região, o que não contribui para uma estabilização de tronco e, assim, pode, até mesmo, aumentar a assimetria postural desses sujeitos. Na literatura, é descrito que a ativação anormal da musculatura é um dos fatores agravantes das alterações posturais
Tabela 4: Média do RMS normalizado como cavalo parado no início e no fim da sessão

\begin{tabular}{|c|c|c|c|}
\hline refa & Condição & Músculos & Média do RMS normalizado \\
\hline \multirow[t]{8}{*}{1} & 1 & TSE & 1,43 \\
\hline & & TSD & 1,08 \\
\hline & & ME & 0,49 \\
\hline & & $\mathrm{MD}$ & 0,58 \\
\hline & & $A B E$ & 0,41 \\
\hline & & $A B B$ & 0,36 \\
\hline & & PVE & 0,46 \\
\hline & & PVD & 0,39 \\
\hline \multirow[t]{8}{*}{1} & 2 & TSE & 1,07 \\
\hline & & TSD & 0,58 \\
\hline & & $\mathrm{ME}$ & 0,36 \\
\hline & & $\mathrm{MD}$ & 0,45 \\
\hline & & $A B E$ & 0,44 \\
\hline & & $A B B$ & 0,31 \\
\hline & & PVE & 0,82 \\
\hline & & PVD & 0,41 \\
\hline \multirow[t]{8}{*}{1} & 3 & TSE & 0,49 \\
\hline & & TSD & 0,63 \\
\hline & & $\mathrm{ME}$ & 0,39 \\
\hline & & $M D$ & 0,54 \\
\hline & & $A B E$ & 0,89 \\
\hline & & $A B B$ & 0,47 \\
\hline & & PVE & 0,47 \\
\hline & & PVD & 0,68 \\
\hline \multirow[t]{8}{*}{1} & 4 & TSE & 0,36 \\
\hline & & TSD & 0,36 \\
\hline & & $\mathrm{ME}$ & 0,37 \\
\hline & & MD & 0,52 \\
\hline & & $A B E$ & 0,60 \\
\hline & & $A B B$ & 0,50 \\
\hline & & PVE & 0,49 \\
\hline & & PVD & 0,33 \\
\hline \multirow[t]{8}{*}{2} & 1 & TSE & 0,73 \\
\hline & & TSD & 0,60 \\
\hline & & $\mathrm{ME}$ & 0,55 \\
\hline & & $M D$ & 0,62 \\
\hline & & $A B E$ & 0,46 \\
\hline & & $A B B$ & 0,42 \\
\hline & & PVE & 0,49 \\
\hline & & PVD & 0,51 \\
\hline \multirow[t]{8}{*}{2} & 2 & TSE & 0,59 \\
\hline & & TSD & 0,32 \\
\hline & & $\mathrm{ME}$ & 0,41 \\
\hline & & $M D$ & 0,45 \\
\hline & & $A B E$ & 0,41 \\
\hline & & $A B B$ & 0,29 \\
\hline & & PVE & 0,84 \\
\hline & & PVD & 0,37 \\
\hline \multirow[t]{8}{*}{2} & 3 & TSE & 0,55 \\
\hline & & TSD & 0,99 \\
\hline & & $\mathrm{ME}$ & 0,44 \\
\hline & & $\mathrm{MD}$ & 0,58 \\
\hline & & $A B E$ & 0,97 \\
\hline & & $A B B$ & 0,57 \\
\hline & & PVE & 0,62 \\
\hline & & PVD & 1,03 \\
\hline \multirow[t]{8}{*}{2} & 4 & TSE & 0,36 \\
\hline & & TSD & 0,40 \\
\hline & & ME & 0,73 \\
\hline & & $\mathrm{MD}$ & 0,52 \\
\hline & & $A B E$ & 0,64 \\
\hline & & $A B B$ & 0,64 \\
\hline & & PVE & 0,30 \\
\hline & & PVD & 0,40 \\
\hline
\end{tabular}

Tarefa 1, sentado inicial; Tarefa 2, sentado final; Condição 1, manta com pé no estribo; Condição 2, manta com pé fora do estribo; 3 . Sela com pé no estribo; 4 . Sela com pé fora do estribo; Músculos: TSE: Trapézio/fibras superiores, lado esquerdo; TSD: Trapézio/fibras superiores, lado direito; ME: Multífido, lado esquerdo; MD: Multífido, lado direito; ABE: Reto abdominal, lado esquerdo; ABD: Reto abdominal, lado direito; PVE:

Paravertebral, lado esquerdo; PVD: Paravertebral, lado direito. 
Tabela 5: Média do RMS normalizado durante as sessões

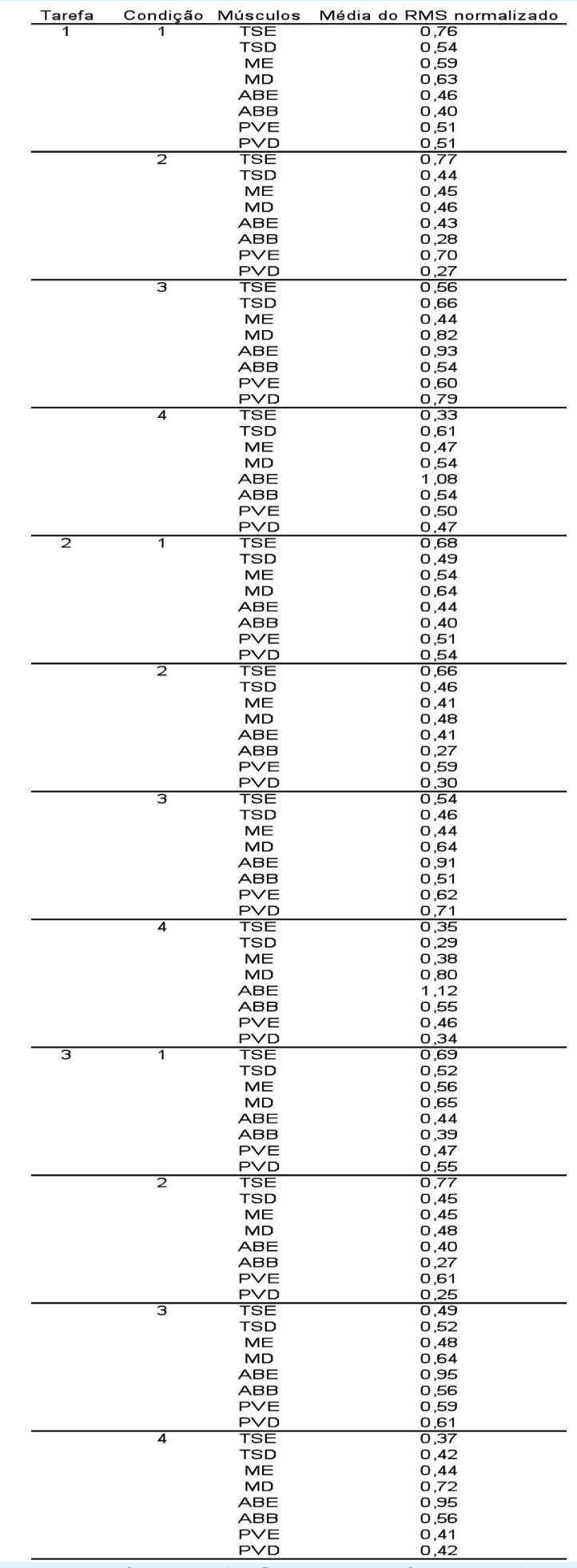

Momentos: 1. Coleta, no 1'; 2. Coleta, no 15'; 3. Coleta, no 30'. Condição 1, manta com pé no estribo; Condição 2, manta com pé fora do estribo; 3 . Sela, com pé no estribo; 4 . Sela, com pé fora do estribo: Músculos: TSE: Trapézio/fibras superiores, lado esquerdo; TSD: Trapézio/fibras superiores, lado direito; ME: Multífido, lado esquerdo; MD: Multífido, lado direito; ABE: Reto abdominal, lado esquerdo; ABD: Reto abdominal, lado direito; PVE: Paravertebral, lado esquerdo; PVD: Paravertebral, lado direito.
Figura 2: Média do RMS normalizado na condição sela com pé no estribo nos momentos 1,2 e 3 (Ml, M2 e M3)

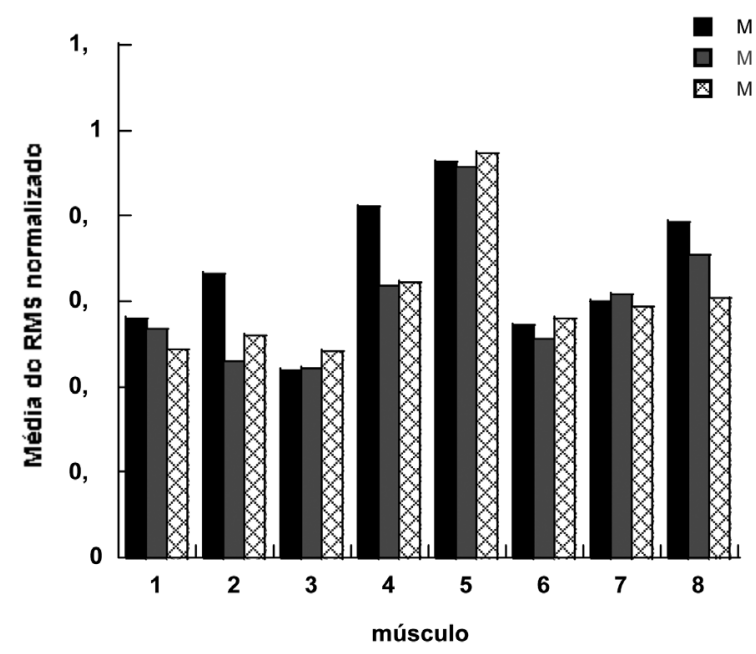

Os números representam os músculos: TSE: Trapézio/fibras superiores, lado esquerdo; TSD: Trapézio/fibras superiores, lado direito; ME: Multífido, lado esquerdo; MD: Multífido, lado direito; $A B E$ : Reto abdominal, lado esquerdo; $A B D$ : Reto abdominal, lado direito; PVE: Paravertebral, lado esquerdo; PVD: Paravertebral, lado direito.

Figura 3: Média do RMS normalizado na condição sela com pé fora do estribo nos momentos 1, 2 e 3 (M1, M2 e M3)

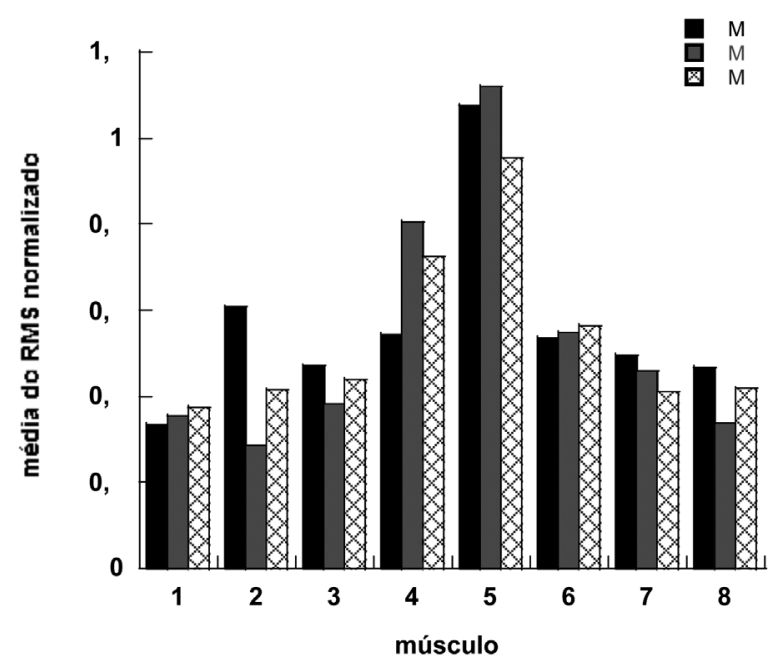

Os números representam os músculos: TSE: Trapézio/fibras superiores, lado esquerdo; TSD: Trapézio/fibras superiores, lado direito; ME: Multífido, lado esquerdo; MD: Multífido, lado direito; $A B E$ : Reto abdominal, lado esquerdo; $A B D$ : Reto abdominal, lado direito; PVE: Paravertebral, lado esquerdo; PVD: Paravertebral, lado direito. 
Figura 4: Média do RMS normalizado na condição sela com pé no estribo nas tarefas sentado inicial (SI) e sentado final (SF)

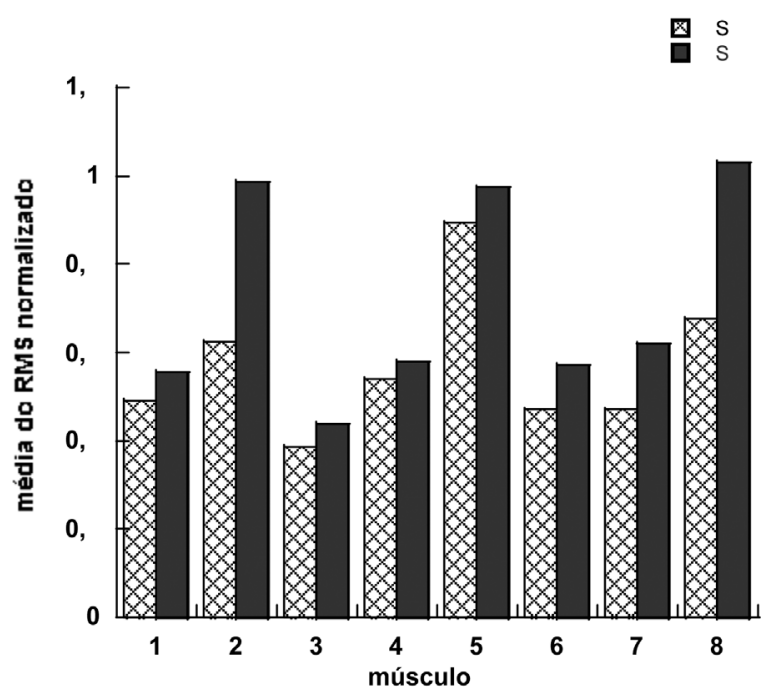

Os números representam os músculos: TSE: Trapézio/fibras superiores, lado esquerdo; TSD: Trapézio/fibras superiores, lado direito; ME: Multífido, lado esquerdo; MD: Multífido, lado direito; $A B E$ : Reto abdominal, lado esquerdo; ABD: Reto abdominal, lado direito; PVE: Paravertebral, lado esquerdo; PVD: Paravertebral, lado direito.

dessa população ${ }^{26}$. A partir desse dado, optouse por analisar somente as condições em que a sela foi utilizada.

Durante a sessão, houve grande variabilidade na ativação muscular ao longo do tempo (M1 a M3) em todos os músculos, justificando, assim, a contribuição do movimento tridimensional no controle da atividade muscular. Conforme evidências nas pesquisas, em $30 \mathrm{mi}$ nutos de montaria a passo, os movimentos proporcionados pelo cavalo promovem em média 30 mil ajustes tônicos no corpo do praticante. Tais movimentos deslocam o corpo do indivíduo de seu centro de gravidade e, desse modo, para que ele consiga permanecer sobre o cavalo, a participação do corpo inteiro é exigida ${ }^{27}$.

Outro achado interessante deste estudo foi uma maior ativação no músculo reto abdominal esquerdo e multífido nas duas condições em que a sela foi utilizada, às vezes com maior atividade do que a registrada na CVM. Além de todos os músculos estarem mais ativos após uma sessão, houve também maior contribuição dos músculos essenciais para o controle do tronco. Este é um dado importante para o tratamento do desalinhamento e dos déficits de equilíbrio comuns na hemiparesia espástica. Essa ativação mais homogênea dos músculos estudados pode garantir um melhor alinhamento postural, uma vez que a alteração na postura está relacionada à dificuldade de ativação da musculatura de forma adequada. Isso, por sua vez, pode acarretar alterações na funcionalidade da postura e marcha, prejudicando o desenvolvimento ${ }^{28-30}$. Além disso, é descrito na literatura que a ativação tônica muscular dos músculos abdominais e das costas, acima dos níveis de repouso, está presente durante a postura sentada ereta, o que sugere que certo grau de ativação muscular é necessário para apoiar a coluna e gerar estabilidade postural. E ainda, que há um aumento na ativação muscular do tronco quando é provocada uma oscilação, sugerindo que os níveis tônicos de ativação muscular fornecem apenas uma margem limitada de estabilidade, enquanto a perturbação, seguindo a estabilidade postural, requer uma resposta dinâmica específica da musculatura do tronco ${ }^{31}$.

Comparando as tarefas SI e SF, nota-se que, após a sessão, houve um aumento na variabilidade da ativação muscular, ou seja, em SI predominava uma maior atividade na região cervical, às vezes até maior do que os valores registrados na CVM. Na tarefa SF, verificou-se um aumento da atividade nas regiões lombar $\mathrm{D}$, abdominal $\mathrm{D}$ e E e torácica D e E. Isso se justifica pelo fato de o cavalo nunca permanecer totalmente parado, pois a troca de apoio das patas, o deslocamento da cabeça ao olhar para os lados, as flexões da coluna, o abaixar e alongar do pescoço obrigam o cavaleiro a fazer ajustes na ativação muscular, a fim de responder aos desequilíbrios provocados por esses movimentos ${ }^{32}$.

Algumas limitações podem ser apontadas neste estudo, como o baixo número de sujeitos, que impossibilitou a realização de testes mais precisos. O avaliador não foi cego e não houve aleatorização das sessões com relação aos ma- 
teriais de montaria. Em alguns momentos, observou-se, durante as coletas, ativação muscular maior do que a registrada na CVM para alguns músculos, o que pode estar relacionado à dificuldade de compreensão do teste por parte dos sujeitos estudados. Apesar disso, este trabalho contribui com a literatura relacionada ao tema e auxilia na tomada de decisão na prática clínica.

\section{Conclusão}

Pode-se concluir com este estudo, por meio da eletromiografia, que usar sela com pé no estribo foi a melhor opção para tratamento equoterapêutico nos praticantes com PC espástica hemiparética avaliados. Essa condição garantiu uma ativação muscular mais homogênea nos músculos do tronco. Vale destacar que este é um trabalho inédito sobre a escolha do melhor tipo de montaria por meio da avaliação eletromiográfica da musculatura de crianças com paralisia cerebral submetidas a tratamento equoterapêutico.

\section{Ägradecimentos}

Os autores agradecem o suporte financeiro, para realização desta pesquisa, proporcionado pelas seguintes instituições: Conselho Nacional de Desenvolvimento Científico e Tecnológico (CNPq), Coordenação de Aperfeiçoamento de Pessoal de Nível Superior (CAPES), Fundação de Amparo à Pesquisa do Estado de Minas Gerais (FAPEMIG), Fundação de Ensino e Pesquisa de Uberaba (FUNEPU) e Associação de Pais e Amigos dos Excepcionais de Uberaba (APAE).

\section{Referências}

1. Rosenbaum P, Paneth N, Leviton A, Goldstein M, Bax MA. A report: the definition and classification of cerebral palsy. Dev Med Child Neurol. 2007;49(S109):S8-14.
2. Mancini MC, Alves ACM, Schaper C, Figueredo EM, Sampaio RF, Coelho ZA et al. Gravidade da paralisia cerebral e desempenho funcional. Rev Bras Fisioter. 2004;8(3):253-60.

3. Teixeira-Arroyo C, Oliveira SRG. Atividade aquática e a psicomotricidade de crianças com paralisia cerebral. Motriz Rev Educ Fis.2007 abr/jun;13(2):97-105.

4. Romeo DJMM, Cioni M, Scoto M, Mazzone L, Palermo FE, Romeo M G. Neuromotor development in infants with cerebral palsy investigated by the Hammersmith Infant Neurological Examination during the first year of age. Eur J Paediatric Neurol 2008;12(1):24-31.

5. Scholtes VA, Dallmeijer AJ, Rameckers EA, Verschuren O, Tempelaars E, Hensen M, Becher JG. Lower limb strength training in children with cerebral palsy - a randomized controlled trial protocol for functional strength training based on progressive resistance exercise principles. BMC Pediatrics. 2008;8(41):1471-2431.

6. Crompton J, Galea MPE, Phillips B. Hand-held dynamometry for muscle strength measurement in children with cerebral palsy. Dev Med Child Neurol. 2007;49:106-11.

7. Sá CSC, Santos FH. Mudanças motoras, sensoriais e cognitivas em crianças com paralisia cerebral espástica diparética submetidas à intervenção fisioterapêutica pelas abordagens Kabat ou Bobath. Rev Fisioter Univ São Paulo. 2004;11(1):56-65.

8. Flett PJ. Rehabilitation of spasticity and related problems in childhood cerebral palsy. J Paediatr Child Health. 2003;39:6-14

9. Allegretti AL, Mancini MC, Schwartzman, JS. Estudo do desempenho funcional de crianças com paralisia cerebral diparética espástica utilizando o Pediatric Evaluation of Disability Inventory. Temas Desenvolvimento. 2002;11(64):5-11.

10. Darrah J, Wessel J, Nearingburg P. O'Connor, M. Evaluation of a community fitness program for adolescents with cerebral palsy. Pediatric Phys Ther.1999;11:18-23.

11. Souza AM, Ferrareto I. Paralisia cerebral - aspectos práticos. São Paulo: Memnon; 1998.

12. Volpon JB. Avaliação e princípios de tratamento ortopédico do paciente com seqüela de paralisia cerebral. Acta Ortop Bras. 1997;5(1). 
13. Damiano DL, Quinlivan BF, Owen M, Shaffrey M, Abel MF. Spasticity versus strength in cerebral palsy: relationships among involuntary resistance, voluntary torque, and motor function. Eur J Neurol. 2001;8(5):40-9.

14. Rotta NT. Paralisia cerebral, novas perspectivas terapêuticas. Jornal de Pediatria. 2002;78(1).

15. Lianza S, Pavan K, Lourenço AF, Fonseca AP, Leitão AV, Musse CAI, et al. Análise epidemiológica de Consenso Nacional sobre espasticidade. Med Reabil.2002;58:2-5.

16. Mewasingh LD, Sékhara T, Pele K, Missa AM, Cheron G, Dan B. Motor strategies in standing up in children with hemiplegia. Pediatr Neurol. 2004;30(4):257-61.

17. Tachdjian MO. Ortopedia pediátrica. $2^{a}$ ed. São Paulo: Manole; 1995.

18. Copetti F, Mota CB, Graup S, Menezes KM, Venturini EB. Comportamento angular do andar de crianças com Síndrome de Down após intervenção com equoterapia. Rev Bras Fisioter. 2007:11(6).

19. Espindula AP, Fernandes M, Ferreira AA, Ferraz MLF, Cavellani CL, Ferraz P F, et al. Flexibilidade muscular em indivíduos com deficiência intelectual submetidos à equoterapia: estudo de casos. Rev Ciênc Ext. 2012;8(2):125-133.

20. Bertoti DB. Effect of therapeutic horseback riding on posture in children with cerebral palsy. Phys Ther. 1988 Oct;68(10):1505-12.

21. Shinomiya $Y$, Ozawa T, Hosaka Y, Wang S, Ishida $\mathrm{K}$, Kimura T. Development and physical training evaluation of horseback riding therapeutic equipment. Proceedings of the 2003 IEEU/ASME International Conference on Advanced Intelligent Mechatronics (AIM 2003). 2003;2:1239-43.

22. Sterba JA, Rogers BT, France AP, Vokes DA. Horseback riding in children with cerebral palsy: effect on gross motor function. Dev Med Child Neurol. 2002;44:301-8.
23. Cherng R, Liao H, Leung HWC, Hwang A. The effectiveness of therapeutic horseback riding in children with spastic cerebral palsy. Adapt Physactiv. 2004;21(2):103-21

24. Hermens JH, Freriks B, Klug CD, Rau G. Development of recommendations for SEMG sensors and sensor placement procedures. J Electromyogr Kinesiol. 2000;14:361-74.

25. Surface Electromyography for Non-Invasive Assessment of Muscle - SENIAM [internet], Netherlands. Disponível em: www.seniam.org.br

26. Ohata K, Tsuboyama T, Haruta T, Ichihashi N, Kato T, Nakamura T. Relation between muscle thickness, spasticity, and activity limitations in children and adolescents with cerebral palsy. Dev Med Child Neurol. 2008;50:152-6.

27. Calill F. Curso básico de equoterapia. In: Congresso Internacional de Paralisia Cerebral. Goiânia; 2004.

28. Porter D, Michael S, Kirkwood C. Patterns of postural deformity in non-ambulant people with cerebral palsy: what is the relationship between the direction of scoliosis, direction of pelvic obliquity, direction of windswept hip deformity and side of hip dislocation? Clin Rehabil. 2007;21(12):1087-96.

29. Koman LA, Smith BP, Shilt JS. Cerebral palsy. Lancet. 2004;363(15):1619-31.

30. Holmes KJ, Michael SM, Thorpe SL, Solomonidis SE. Management of scoliosis with special seating for the non-ambulant spastic cerebral palsy population: a biomechanical study. Clin Biomech. 2003;18:480-7.

31. Masani K, Sin VW, Vette AH, Thrasher TA, Kawashima N, Morris A, et al. Postural reactions of the trunk muscles to multi-directional perturbations in sitting. Clinical Biomechanics. 2009;24:176-82.

32. Ande-Brasil. Apostila do curso básico de equoterapia. Brasília; 2011. 